C-A/AP/\#139

February 2004

$\underline{\text { eRHIC Pressure Rise and Electron Cloud }}$

S.Y. Zhang

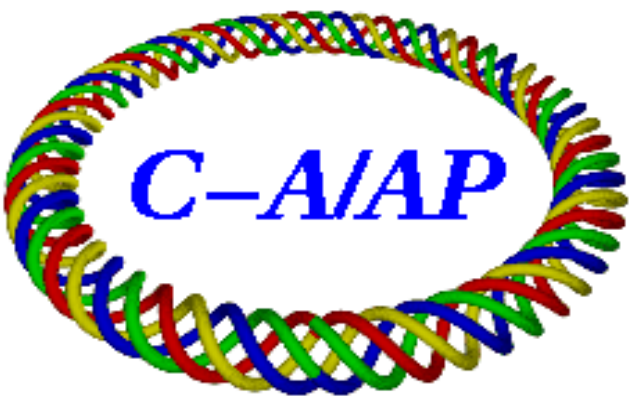

Collider-Accelerator Department Brookhaven National Laboratory

Upton, NY 11973 


\title{
eRHIC Pressure Rise and Electron Cloud
}

\author{
S.Y. Zhang
}

February 26, 2004

\begin{abstract}
Current RHIC pressure rise and electron cloud are briefly reviewed. In the eRHIC scenario with 360 bunches in the ring, the electron cloud may take place at the cold region, and at the beam store as well. Plan of counteract is discussed.
\end{abstract}

\section{Injection pressure rise}

Pressure rise at the injection has been observed for gold, deuteron, and proton operations in the RHIC. This pressure rise limits operation of 112 bunches with bunch intensity of $10^{9}$ gold ions, and $10^{11}$ for protons [1].

It has been diagnosed that the injection pressure rise is due to the electron multipacting, i.e. electron cloud. The evidences include,

1. Electron detector signals are very closely related with the pressure rise, at onset, saturation, and drooping.

2. Pressure rise and electron signal are very sensitive to the bunch spacing, 112 bunch mode is much worse than 56 bunch mode. Note that the sensitivity to bunch spacing is an important characteristic of the electron cloud.

3. Bunch gap helps.

4. Solenoid field of 5 to 50 Gauss can suppress both pressure rise and electron signal, but not completely. 
5. Beam scrubbing has been demonstrated helpful in reducing pressure rise.

On the other hand, the electron cloud observed at the RHIC is different from other machines. For example, the RHIC electron cloud takes place at the bunch spacing of 108 ns or even 216 ns. All other machines have much smaller bunch spacing.

1. The B factories, KEKB and PEPII, have bunch spacing of 4 ns to 8 ns.

2. Electron cloud was peaked at 20 ns of bunch spacing at the APS of Argonne.

3. SPS observed electron cloud at $25 \mathrm{~ns}$ bunch spacing with the threshold of bunch intensity of 3 to $4 \times 10^{10}$ protons. At the bunch spacing $130 \mathrm{~ns}$, no electron cloud observed at the bunch intensity of $2.5 \times 10^{11}$ protons.

4. Tevatron observed electron cloud at 18.9 ns bunch spacing with bunch intensity of $4 \times 10^{10}$ protons. The situation is very similar to SPS. The Tevatron Run II plan calls for 132 ns bunch spacing with bunch intensity of $2.7 \times 10^{11}$ protons.

The RHIC pressure rise and electron cloud have several distinguished characteristics from other machines.

1. It only takes place in warm sections, and the pressure rise distribution in the ring is very un-uniform. When pressure rise at certain location(s) is high enough to close the vacuum valve, many locations have none. The worst locations also may change.

2. Given same chambers, the beam intensity threshold at the Q3 to Q4 straight section, 34 meters long, is only $60 \%$ of that at the interaction straight section, which is 17 meters long.

3. No noticeable cryogenic heat load has been observed. Together with the absence of electron cloud induced beam instability and emittance growth, it is believed there is no electron multipacting at the RHIC cold region. 

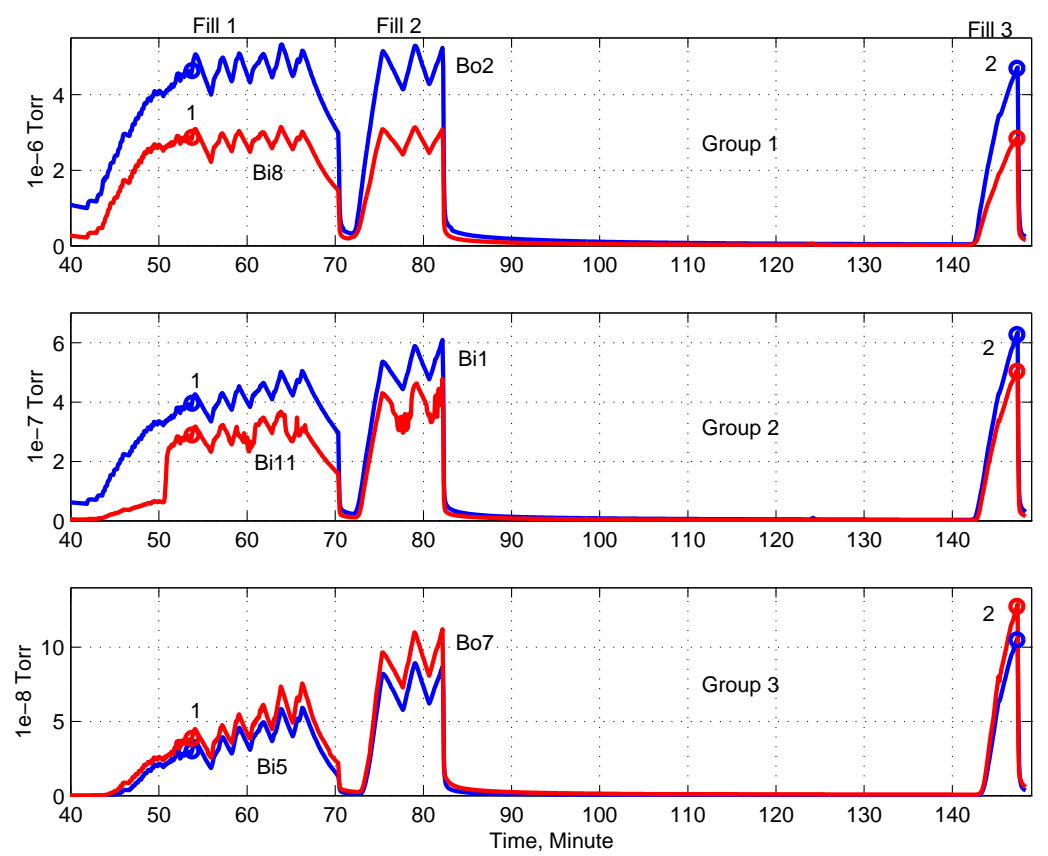

Figure 1: Typical pressure rise pattern for three different groups, with the high, medium, and low pressure rises.

4. RHIC pressure rise decreases at the ramp, and it is non-existent at the store. In SPS, the electron activity was stronger at the store than at the injection [2].

It is suspected that the beam halo scraping at the wall, which generates mostly positive ions, may have helped the secondary electron to survive long bunch gap, and makes electron multipacting possible. If this is the case, then most RHIC injection pressure rise observations can be explained.

During the 2003 polarized proton run, a beam scrubbing was studied. Total high intensity beam scrubbing time was less than 1 hours. However, beam scrubbing effect was observed not only in the locations with highest pressure rise, but also in others with non-trivial pressure rise.

In Fig.1, it is shown that for locations with high pressure rise at about $5 \times 10^{-6}$ Torr, the pressure rise kept about the same for 3 fills. For locations with medium pressure rise of about $5 \times 10^{-7}$ Torr, the pressure rise increased. For locations with low pressure rise at less than $5 \times 10^{-8}$ Torr, the pressure rise of $3 \mathrm{rd}$ fill is about 2.5 times higher than the first fill, which is approximately 


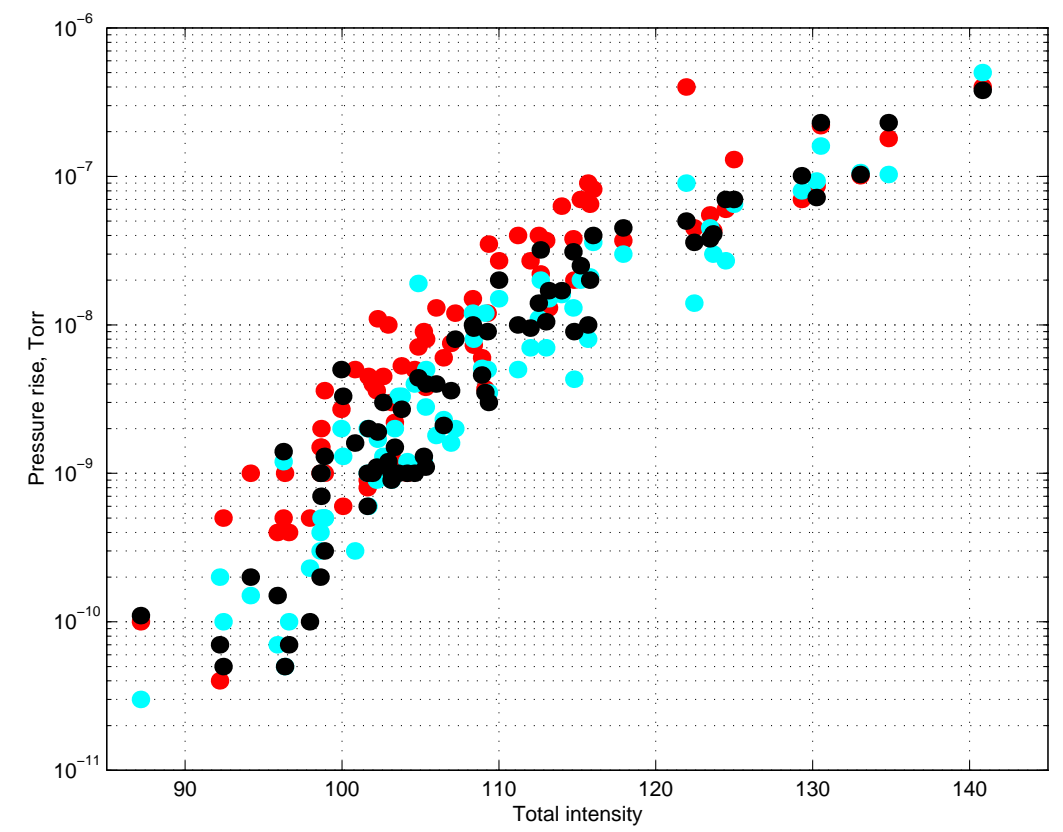

Figure 2: Transition pressure rise at IR2 (red), IR10 (cyan), IR12 (black). Intensity unit is charge equivalent to $1 \mathrm{e} 9 \mathrm{Au}$ ions.

reflecting the beam strength in terms of exciting electron multipacting.

For RHIC operation, the complete elimination of the injection pressure rise is not necessary. Therefore, a limited time of high intensity beam run might be sufficient, and it is feasible of applying the beam scrubbing to allow higher beam intensity operation.

\section{Transition pressure rise}

The beam transition pressure rise for heavy ion operations is another intensity limit for the RHIC. In Fig.2, the transition pressure rises in the deuterongold (d-Au) run at IR2(BRAHMS), IR10(PHOBOS), and IR12 are shown against the total beam intensity.

The characteristics of this pressure rise are as follows.

1. The pressure rise is quasi-exponentially proportional to the total beam (charge) intensity. 
2. For same intensity, no difference between 56 bunch and 112 bunch modes can be identified. The absence of bunch spacing effect indicates that the transition pressure rise is not dominated by the electron cloud. Another evidence is that no electron multipacting signal has been detected at the transition.

3. In high intensity ramps shown in Fig.2, the beam loss at the transition varies from $1 \%$ to larger than $10 \%$. The reasonably narrow band in the pressure rise distribution indicates that the beam loss is not a dominant factor.

4. The pressure rise is not related to the ion species. The gold beam intensity was considerably higher than the deuteron in early run. After the deuteron bunch merge in the AGS Booster, the deuteron intensity was much higher than gold beam. No difference can be identified regarding to these different beams. Since the gold ion gas desorption cross section is about 79 times larger than the deuteron ion, this indicates that the gas desorption is not a dominant factor in the transition pressure rise.

It is found that the transition pressure rise is, on the other hand, related to the beam momentum spread.

1. The beam momentum spread is $0.17 \%$ at the injection, it is peaked at $0.3 \%$ at the transition, and decreases afterwards. The pressure rise follows this pattern. In proton run, the beam momentum spread decreases in the acceleration, and the pressure rise decreases as well.

2. The bunch length seems not a dominant factor in the transition pressure rise. At the beam rebucketing, where the bunch was captured in 200 $\mathrm{MHz}$ storage cavity, the bunch length reduces to $5 \mathrm{~ns}$, the same as that at the transition (the beam potential at the rebucketing is actually $37 \%$ higher than that at the transition due to the smaller transverse size), yet no rebucketing pressure rise observed in the d-Au run.

3. The total storage cavity voltage was $2.5 \mathrm{MV}$ in $\mathrm{d}-\mathrm{Au}$ run. At the rebucketing, the beam momentum spread was $0.17 \%$, the same as that at the injection, and much lower than $0.3 \%$ at the transition. This may explain the absence of the pressure rise at the rebucketing in $\mathrm{d}-\mathrm{Au}$ run. 
In Run 4, two more common cavities have been commissioned to increase the total rebucketing voltage to higher than $4 \mathrm{MV}$. The beam momentum spread at the rebucketing becomes larger than $0.19 \%$. The pressure rise was observed at several interaction regions. Much higher storage voltage and the better rebucketing imply also higher beam peak current and beam potential. Accordingly, some electron multipacting have been observed at the rebucketing in Run 4.

The transition pressure rise had caused serious experiment background problem in d-Au run. For same luminosity, 56 bunch mode requires $30 \%$ less total intensity than the 112 bunch mode. Switching from 112 bunch mode to 56 bunch, the experiment background was significantly improved. One might expect that the background problem will be relevant again at higher beam intensity, since given 56 bunch mode, the luminosity increases at the square of the bunch intensity, whereas the transition pressure rise is quasi-exponential to that.

The transition pressure rise is currently a show stopper for 112 bunch operation. In Run 4, 56 bunch mode is adopted.

Some NEG (non-evaporable-getter) pipes have been installed in the RHIC rings for test. The roughness of the activated NEG coating surface is essential for better pumping. As by-products, the SEY (secondary electron yield) and electron desorption reductions have been measured for the NEG coating, where the rough surface may have contributed. To alleviate the transition pressure rise, ion desorption reduction might be more important. The data on this aspect is, however, less than sufficient. The NEG pipes in RHIC have made possible for the evaluation on ion desorption, and also for other issues, such as the activation condition, the saturation effect, the aging, venting effect, possible dust, and impedance problem, etc.

For same purpose, a test stand has been built at the Tandem Van de Graaff. Different activations will be tested, and also the ion desorption on the shallow angle ion beam scraping on stainless steel and NEG surface will be compared.

\section{Scenario of 360 bunches in RHIC}

In the scenario of 360 bunches in the RHIC, not only the injection and transition pressure rises, but also a usual electron cloud may take place for both proton and heavy ion beams. With the bunch spacing of $35 \mathrm{~ns}$, eRHIC 
is very similar to SPS and LHC in terms of electron multipacting. In Table 1, the eRHIC heavy ion and proton parameters are compared with the SPS and LHC, where $\langle\Delta E\rangle$ is the energy gained by electrons per bunch passing.

\begin{tabular}{|ccccc|}
\hline Injection/store & eRHIC, Au & eRHIC, p & SPS & LHC \\
\hline Kinetic energy, $E_{k}, G e V / u$ & $8.9 / 100$ & $24 / 250$ & $26 / 450$ & $450 / 7000$ \\
Bunch intensity, $N_{b h}, 10^{11}$ & 0.79 & 1 & 1 & 1 \\
Bunch spacing, $t_{b s}, n s$ & 35 & 35 & 25 & 25 \\
Chamber radius, $b, \mathrm{~cm}$ & 3.45 & 3.45 & 2.5 & 1.74 \\
Beam radius, $a, \mathrm{~mm}$ & $3.0 / 0.93$ & $1.9 / 0.59$ & $3.2 / 0.78$ & $1.15 / 0.293$ \\
Energy gain, $\langle\Delta E\rangle, \mathrm{eV}$ & $104 / 154$ & $198 / 277$ & $267 / 450$ & $728 / 1095$ \\
\hline
\end{tabular}

Table 1

The bunch spacing is one of the most important parameters. In general, larger the bunch spacing, less the secondary electrons survive the bunch gap, and higher SEY is needed for electron multipacting. The 35 ns bunch spacing at the eRHIC is only a little larger than the $25 \mathrm{~ns}$ at SPS and LHC.

The second most important parameter is the energy the electrons gained during the one bunch passing, which is

$$
\langle\Delta E\rangle=\frac{e}{2 m_{e}}\left(\frac{N_{b h} e Z_{0}}{2 \pi b}\right)^{2} \ln \left(\frac{b}{a}\right)
$$

where $Z_{0}=377 \Omega$ is the impedance in free space, and $m_{e}$ is the mass of electron. Most important factor affecting $\langle\Delta E\rangle$ is the bunch intensity.

The intensity threshold at the SPS was considerably lower than the LHC beam requirement. It was 5 to $6 \times 10^{10}$ at the straight sections, and 3 to $4 \times 10^{10}$ at the dipoles. Only after several days of beam scrubbing, the LHC beam requirement was achieved.

For normal electron cloud, eRHIC have several new issues to deal with.

1. Electron multipacting in cold region. The chamber radius at the cold region is $3.46 \mathrm{~cm}$, compared with $6.1 \mathrm{~cm}$ at warm sections. The multipacting threshold at the cold region is, therefore, lower. Once electron cloud built up, the cryogenic heat load will be of concern. Experiment data at the SPS shows that the heat load is larger than $1.2 \mathrm{~W} / \mathrm{m}$ under electron multipacting [3], which is not acceptable for RHIC cryogenic system. 
2. Since the cold region consists $3 / 4$ of the RHIC ring, the electron cloud induced beam instability and beam emittance will be of concern.

3. Electron cloud will present not only at the injection, but also at the ramp and storage.

4. Electron activity in dipole and quadrupole becomes relevant. The multipacting threshold at the dipoles is lower than that at the straight sections. Moreover, since the electron dose stripes in dipoles vary according to the bending field and beam intensity [2], the scrubbing is

more difficult. As for quadrupole field, it is suspected the electrons are trapped there and stay for a long time.

The pressure rise at the cold region is probably not a problem, thanks the cryogenic pumping.

\section{$4 \quad$ Plans}

\subsection{Collaboration with RHIC pressure rise studies}

Active study is undergoing at the RHIC in searching for the pressure rise and electron cloud remedies. Collaboration items with the eRHIC effort include,

1. Beam scrubbing, which has been demonstrated in principle, but further study is needed for incorporating it to the operation. First for proton beam, then for heavy ion beam.

2. Beam scraping study of ion desorption. The ion desorption of high energy particle at glancing angles is still a pending issue. At the RHIC Run 4, warm dipoles will be used to actually scraping ions at the wall to measure the ion desorption rate at the incident angle of a few mrad. Measurement will take place for both stainless steel and NEG pipes for comparison.

3. The relation between the beam momentum spread and the transition pressure rise remains to be clarified. By changing the RF voltage at the transition, this can be studied. The approach has a potential to provide a remedy for this pressure rise. 
The RHIC effort in reducing the beam induced pressure rise is past two years has already gained much better understanding and the machine improvement. These efforts, such as baking, solenoid, beam scrubbing, beam injection pattern, and NEG coating will likely to take effect and gradually improve the RHIC performance.

\subsection{Collaboration with LHC-SPS electron cloud stud- ies}

The 360 bunches mode cannot be studied at the RHIC, due to the lack of beam injection apparatus. Since the situation will be very similar to the LHC, a collaboration should be pushed forward. Fortunately the intense studies have been undergoing for several years in the CERN, including numerous beam experiments at the SPS, simulation, and theoretical effort. Suggested collaboration items include,

1. Heat load problem. Experiments at the SPS during the 2003 run have shown that the heat load is significant enough to be treated seriously. Further data are of interest.

2. Electron activity at dipoles and quadrupoles.

3. Beam scrubbing effect at the cold region. Also in 2003 SPS experiment, it was found that the cold region scrubbing was much less effective than that at the warm region [3].

According to these results, the current LHC plan calls for initially using not higher than $4 \times 10^{10}$ protons per bunch for $25 \mathrm{~ns}$ bunch spacing, and/or a 75 ns bunch spacing injection. The LHC scrubbing scenario is under study, which is pending on several unknowns, such as the possible beam instability during the scrubbing, the tolerable maximum heat load, and magnet quenching problem.

The eRHIC-LHC collaboration should be on both experimental and theoretical aspects, and simulation will be an important tool.

\subsection{0 bunch scenario}

Given luminosity unchanged, it is of interest to study the benefit of using

larger bunch spacing and higher bunch intensity. 
Issues related with the 180 bunch mode, with the 70 ns bunch spacing and $40 \%$ increase in bunch intensity, include,

1. Electron activity will be reduced compared with the 360 bunch mode. The decrease of the electron activity is more than linearly proportional to the inverse of bunch spacing. The experimental data of the SPS are shown in Table 2 [4].

\begin{tabular}{|ccccc|}
\hline Bunch spacing & 25 & 50 & 75 & $n s$ \\
Bunch intensity threshold & 0.3 & 0.6 & 1.2 & $10^{11}$ \\
\hline
\end{tabular}

Table 2

2 In 180 bunch scenario, the total beam intensity is reduced. This will benefit at least the pressure rise in warm sections, perhaps more.

3 Heat load needs to be estimated, including the resistive wall contribution.

4 Beam scrubbing needs to be studied.

\section{References}

[1] S.Y. Zhang et al, PAC, 2003, Portland, Oregon.

[2] J.M. Jimenez, Pressure rise workshop. Dec. 2003, BNL.

[3] F. Ruggiero, Pressure rise workshop. Dec. 2003, BNL.

[4] G. Arduini, Chamonix XIII, Jan. 2004. 